\title{
Parâmetros Alimentares de Hoplias malabaricus, como ferramenta de análise Ambiental na Lagoa Feia, RJ, Brasil.
}

\section{Food Parameters Hoplias malabaricus as Environmental Analysis Tool in Lake Ugly, RJ, Brazil.}

\author{
Glieber Henriques Beliene ${ }^{1}$, Adélia Rangel Mandu Rocha² ${ }^{2}$ Cristina Maria Magalhães \\ de Souza ${ }^{3}$
}

\author{
${ }^{1}$ Universidade Federal de Mato Grosso - UFMT, Av. Fernando Corrêa da Costa, no 2367, \\ Bairro Boa Esperança, Cuiabá, MT, Brasil. Laboratório de Ecologia Aquática. \\ 23 Universidade Estadual do Norte Fluminense Darcy Ribeiro - UENF, Avenida Alberto \\ Lamego, 2000, Parque Califórnia ,28013-602, Campos dos Goytacazes, RJ, Brasil. Centro de \\ Biociência e Biotecnologia, Laboratório de Ciências Ambientais. \\ guihb bio@yahoo.com.br ${ }^{1}$, adeliarocha1@yahoo.com.br ${ }^{2}$, $^{\text {cristalmmsouza@yahoo.com.br }}{ }^{3}$
}

\begin{abstract}
Recebido:10/ 01/2013, Aceito: 25/01/2013, Publicado: 08/05/2014
RESUMO - Dentre as quase 25.000 espécies de peixes conhecidas no mundo, destaca-se a Hoplias malabaricus (Bloch, 1794) por apresentar características alimentares bem amplas. Trata-se de um peixe caçador de emboscada que por ser carnívoro piscívoro e topo de cadeia, tem se tornado motivo de discussão quanto ao papel que desempenha na regulação de outras populações de peixes. Não se limitando a piscivoria quando jovem também se alimenta de larvas de insetos, e quando adulta complementa sua alimentação com pequenos crustáceos. O objetivo do presente trabalho foi determinar parâmetros alimentares da população de Hoplias malabaricus nos períodos chuvoso e de estiagem na Lagoa Feia no Rio de Janeiro, a fim de se constatar possíveis mudanças no padrão alimentar dessa espécie em função das pressões antrópicas nesse ambiente. Para isso os exemplares de Hoplias malabaricus foram obtidos Novembro/09 e Julho/10. Dos espécimes foram analisados os conteúdos estomacais, e determinados índices de repleção estomacal (IRE) e estados de repleção estomacal (ERE), dos quais os resultados demonstraram majoritariamente a espécie Cyphocharax gilbert como índice alimentar preferencial da Hoplias malabaricus, o que não é comum visto a sua característica amplitude alimentar. Outro fato importante foram os altos índices de captura de alimento mesmo em períodos onde comumente a taxa alimentar da traíra é reduzida. Dessa forma esses indícios apontam para possíveis alterações na dinâmica da comunidade aquática dessa lagoa, certamente em função de perturbações pontuais ou difusas humanas, o que está levando a um aumento na oferta de alimento.
\end{abstract}

Palavras chaves: Traíra, trófico, antrópico, estômago

\begin{abstract}
Among the nearly 25,000 known species of fish in the world, Hoplias malabaricus stands out ( Bloch , 1794 ) for presenting fairly broad food characteristics. This is an ambush fish hunter and for being carnivorous and piscivorous top chain, it has become a matter of debate about the role it plays in regulating other fish populations. Not limited to piscivory when young also feeds on insect larvae, and when adult complements its diet with small crustaceans. The objective of this study was to determine dietary parameters of population of Hoplias malabaricus in the rainy and dry seasons in Lagoa Feia in Rio de Janeiro, in order to observe possible changes in the dietary pattern of the species as a function of anthropogenic pressures in this environment. Some specimen of this Hoplias malabaricus were obtained in November, 9th and July, 10th. From the specimen, stomach contents were analyzed, and indexes repletion ( IRE) and states repletion ( ERE ) were determined, the results of which showed mainly the Cyphocharax gilbert as a preferred food specie index of Hoplias malabaricus, what it is not commom in its characteristic of feed amplitude. Another important fact was the high rates of food capture even in periods when commonly the feed rate of Traíra is reduced. Thus these indications point to possible changes in the dynamics of the aquatic community of the pond, certainly in terms of specific or diffuse human disturbances, that it is leading to an increase in food supply
\end{abstract}

Key words: Traira, trophic, anthropic, stomach

\section{INTRODUÇÃO}

A plasticidade trófica é o termo utilizado para descrever a flexibilidade dos animais em utilizar recursos alimentares disponíveis no ambiente. (Correa \& Piedras 2009), Trata-se de uma característica marcante da ictiofauna tropical sendo especialmente importante em ambientes cuja 
Citação: Beliene G. H., Rocha A. R. M. e Souza C. M. M., Parâmetros Alimentares de Hoplias malabaricus, como ferramenta de análise Ambiental na Lagoa Feia, RJ, Brasil. E\&S - Engineering and Science (2014) ,1:1.

disponibilidade de recursos é variável. Segundo (Martins 2009), dessa forma grande parte das espécies de peixes neotropicais segue o padrão de elevada plasticidade comportamental, em termos alimentares, o que impede que ocorram extinções massivas na maioria dos casos (Martins 2009).

A espécie Hoplias malabaricus, popularmente conhecida como Traíra pertence a família Erythrinidae (Characiformes) que compreende três gêneros: Hoplias, Hoplerythrinus $e$ Erythrinus (Vitorino et al. 2007). Dentre as 12 espécies atualmente conhecidas do gênero Hoplias, a Hoplias malabaricus é a que possui maior distribuição geográfica (Bloch 1794, Penteado 2009). Trata-se de um peixe comum nas águas da América do Sul, ictiofágico, carnívoro (piscívoro quando adulto), complementando sua dieta com insetos (apenas quando jovem), crustácea e outros invertebrados, ocupando o topo a cadeia alimentar (Amaral et al. 2009, Brum et al. 2009, Costa et al. 2009). De costumes sedentários essa espécie é bem adaptada a ambientes lênticos de água doce, com baixa profundidade e com vegetação, onde se refugiam e constroem ninhos, embora possa ser encontrada em rios de pequeno e grande porte (Gimenes 2006).

É uma predadora de tocaia, com estratégia de caça baseada na espreita da presa (Bizerril \& Primo 2001) as quais captura isoladamente ou em grupos, na superfície da água ou meiaágua (Oliveira 1994), preferencialmente durante o período crepuscular (Bizerril \& Primo 2001), e noturno (Penteado 2009).

Devido a sua plasticidade trófica, em períodos de escassez de alimentos a $H$. malabaricus dispõe-se de qualquer recurso disponíveis no ambiente, mostrando sua adaptação a habitats efêmeros (Carvalho et al. 2002). Ela possui um importante papel ecológico na manutenção de comunidades de outras espécies de peixes (Correa \& Piedras 2009), de forma que em lagoas onde a Hoplias malabaricus foi excluída, são significativamente mais percebidas as reduções em riquezas de outras espécies (Petry 2005).

No contexto geral, trata-se de uma espécie que se adapta a diversos habitats (Luiz et al 2008), sendo um bom exemplo de uma espécie amplamente distribuída adequado para estudos em escala continental e biogeográficos, no sul de bacias americana (Jacobina et al. 2009).

O objetivo desse trabalho foi determinar parâmetros alimentares da população de Hoplias malabaricus na Lagoa Feia no Rio de Janeiro, através da análise da dieta por meio de identificação dos itens alimentares, do Estado de Repleção Estomacal (ERE) e do Índice de Repleção Estomacal (IRE), a fim de se observar o comportamento trófico, no ambiente em que vivem, e possíveis pressões antrópicas influenciando esse comportamento. A justificativa de a Lagoa Feia ser escolhida é por se tratar de um local com grande importância para o abastecimento de peixes, para consumo humano, por ser um ambiente de grande importância ecológica e principalmente por ser um local muito pressionado pela ocupação humana ao longo dos tempos. O presente estudo durou oito meses, caracterizando respectivamente as estações de seca e chuva nessa região.

\section{MATERIAL E MÉTODOS}

\section{Área de Estudo}

A bacia hidrográfica da lagoa Feia compreende uma superfície de cerca de $2.900 \mathrm{Km}^{2}$, formada pelos rios Ururaí e Macabu e por uma intricada rede de canais de drenagem e córregos que fluem para a Lagoa Feia. Além disso, a Lagoa Feia é ligada ao mar pelo Canal das Flechas construído pelo Departamento Nacional de Obras de Saneamento (DNOS) em 1949 (Bizerril \& Primo 2001). A fim de aumentar o escoamento de água para o oceano, e com isso diminuir os efeitos das cheias periódicas do rio Paraíba do Sul. Este canal foi responsável pela considerável redução do 
Citação: Beliene G. H., Rocha A. R. M. e Souza C. M. M., Parâmetros Alimentares de Hoplias malabaricus, como ferramenta de análise Ambiental na Lagoa Feia, RJ, Brasil. E\&S - Engineering and Science (2014) ,1:1.

espelho d'água da lagoa que já foi cerca de $112 \mathrm{Km}^{2}$ e atualmente está reduzido aos atuais $12 \mathrm{Km}^{2}$ (Carneiro2004).

A bacia hidrográfica da Lagoa Feia foi formada durante a separação do Continente Sul-americano e a África (ruptura do supercontinente do Sul, Gondwana) durante o Éon cretáceo - Aptiano a cerca de 122 milhões de anos (Gonzaga 2005). A lagoa Feia é, caracterizada por ser uma lagoa de pouca profundidade da coluna d'água com aproximadamente $5 \mathrm{~m}$ (Souza 2006). O clima local pode ser caracterizado como quente úmido com média de $25,3{ }^{\circ} \mathrm{C}$, verificando-se a existência de duas estações distintas no ano: a seca (abril a setembro) e a chuvosa (outubro a março), que juntas caracterizam uma pluviometria média de 71,1 mm (Souza 2006). Ao seu redor estão instalados grandes centros produtivos da região noroeste do Rio de janeiro, destacando-se a Cidade de Campos dos Goytacazes, e Macaé que no último século devido a exploração de petróleo na bacia de Campos, sofreram um inchaço populacional e vem pressionando a qualidade e a quantidade da água desse ambiente dulcícola, comprometendo dessa forma sua integridade ecológica. Além disso, ao longo das últimas décadas, as usinas canavieiras da região vem despejando sem nenhum controle ou fiscalização vinhoto nos afluentes da Lagoa, e que ocorreu um aumento significativo da agropecuária na região(Valpassos \& Neto 2006).

\section{Amostragem}

Os exemplares de Hoplias malabaricus foram obtidos através de compras mensais entre Novembro/09 e Julho/10, com exceção do mês de Janeiro, de forma a se obter representação das duas estações pluviais no local. As compras foram feitas com os pescadores da região que para atividade de captura utilizam redes de espera de diversas malhas. Vinte (20) exemplares de traíras foram obtidos por mês, ao longo de 8 meses de coleta totalizando 160 indivíduos.
A cada compra os exemplares eram levados ao Laboratório de Ciências Ambientais da UENF, a fim de serem devidamente identificados com o mês de coleta, e anotados os valores de PE $=$ Peso do Estômago (g); PT = Peso Total.

Para o estudo da dieta de Hoplias malabaricus os estômagos foram removidos da cavidade abdominal dos indivíduos e preservados em álcool $70 \%$. Posteriormente foram pesados úmidos (PU) e por fim tiveram todo conteúdo estomacal analisado macroscopicamente, e por vezes com auxílio de lupas até a identificação dos itens alimentares até o menor nível taxonômico, baseado na compilação de REIS et al. (2003), além de literatura variada mais específica para peixes neotropicais, e também consulta a especialistas. Para se estabelecer a época em que os animais se apresentaram em melhores condições alimentares, foram estimados o Estado de Repleção Estomacal (ERE), que segundo Santos (1978), se faz analisando o volume ocupado pelos itens alimentares no estômago e classificando-os dentro da seguinte escala: (A) estômago vazio; (B) estômago parcialmente cheio e (C) estômago cheio. $\mathrm{O}$ outro item analisado foi o Índice de Repleção Estomacal (IRE) utilizando-se a fórmula I de Vazoller (1996): $\mathrm{IRE}=(\mathrm{PE} / \mathrm{PT}) \times 100$.

\section{RESULTADOS E DISCUSSÃO}

Os índices de repleção estomacal, (Figura 1) apresentaram valores decrescentes desde o inicio das coletas no mês de novembro, até o mês de abril que marca o início do período sem chuvas na região, seguido por uma elevação no mês de maio e posterior estabilização até o ultimo mês, fato que veio acompanhado pela mesma diminuição dos estados de repleção dos estômagos cheio e semi cheio, mas que quando analisado estatisticamente não denota uma diferença tão significativa (Figura 2). Podemos entender melhor tal resultado estatístico comparando-se as Figuras 1 e 2, onde se observa uma disparidade marcante no mês de junho, quando o percentual de 
Citação: Beliene G. H., Rocha A. R. M. e Souza C. M. M., Parâmetros Alimentares de Hoplias malabaricus, como ferramenta de análise Ambiental na Lagoa Feia, RJ, Brasil. E\&S - Engineering and Science (2014) ,1:1.

repleção dos estômagos cheios e semi cheios forma tão evidente no IRE da população sofre uma redução que não se reflete de como um todo, nesse mesmo mês.

Figura 1 - - Índice médio de repleção estomacal dos exemplares de H. malabaricus entre os períodos de coleta $(\mathrm{N}=160)$.

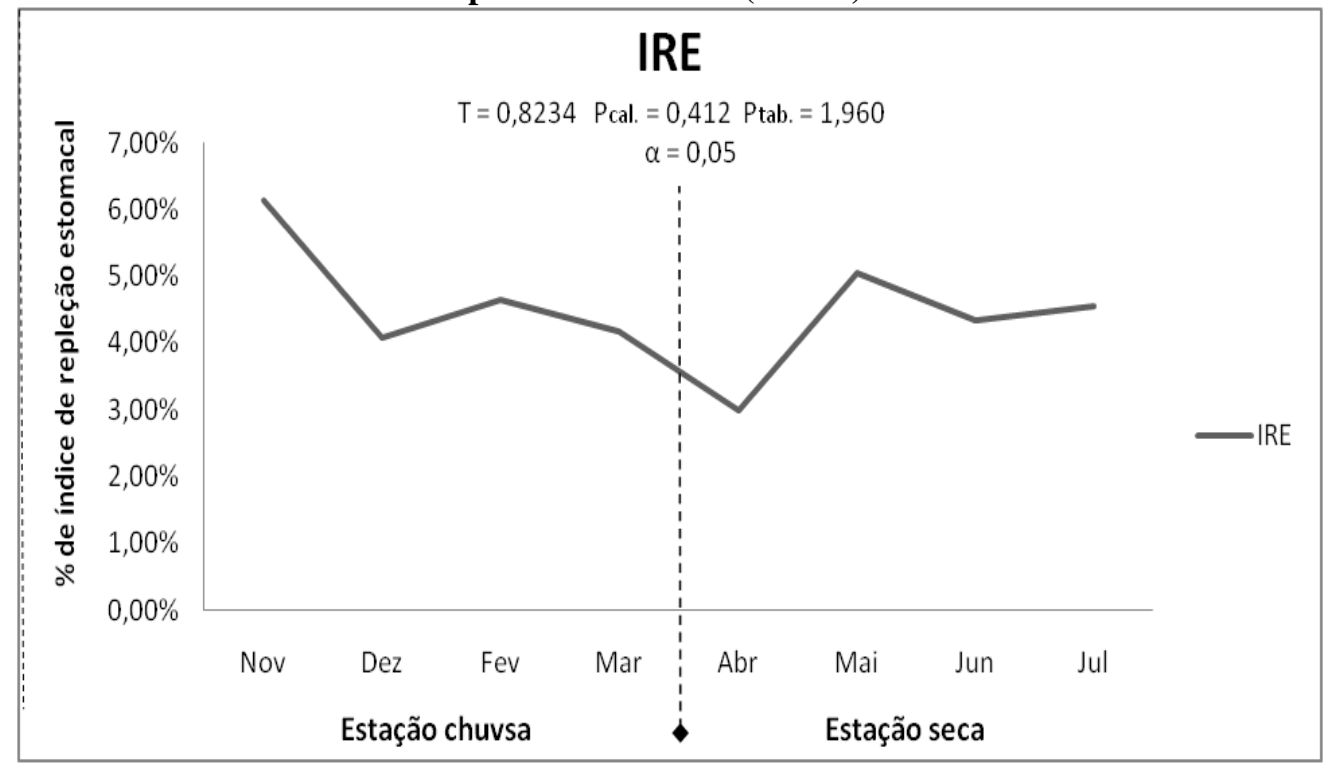

Figura 2 - Percentual dos estados de repleção estomacal cheio e semi cheio no período de coleta para a população de $H$. malabaricus $(N=160)$.

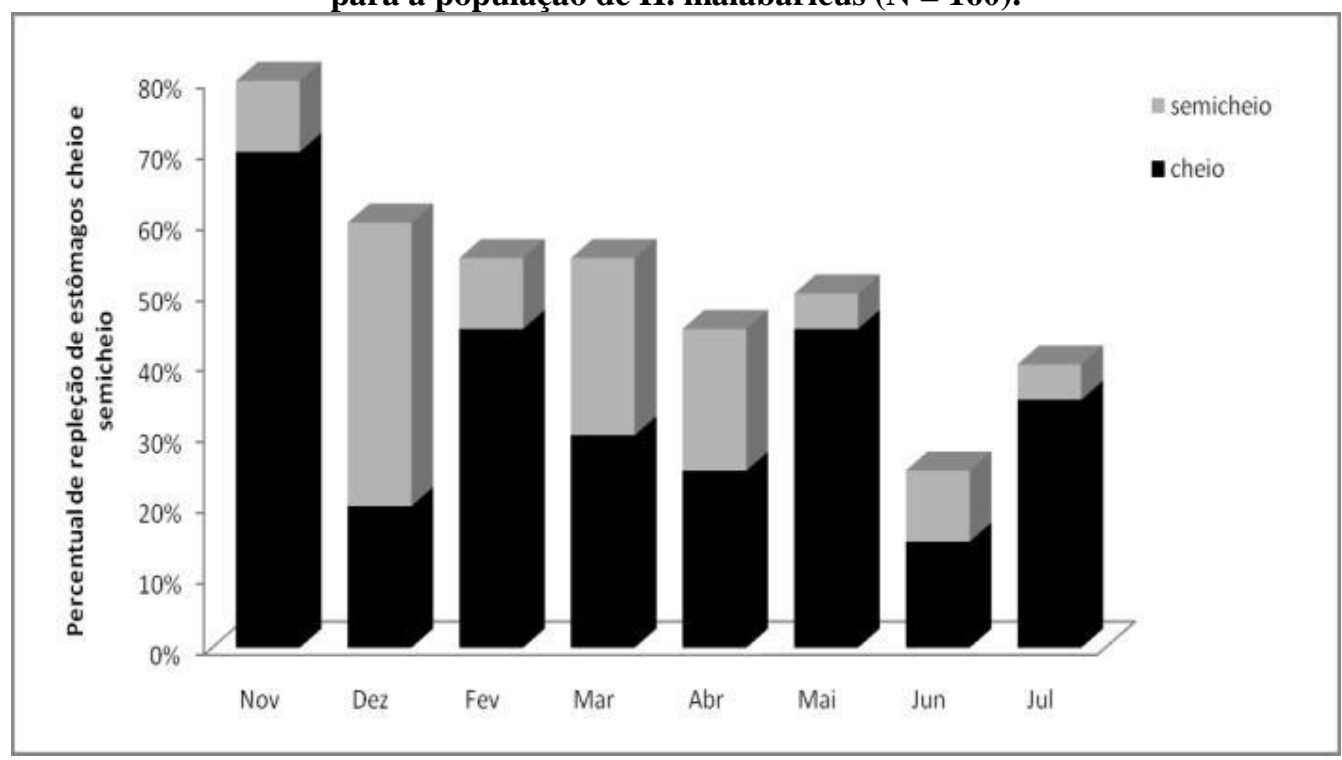

Isso se deve ao fato de que no mês de junho, como mostra a Tab.2 um pequeno número de indivíduos com estômagos cheios e semi cheios conseguiram manter um valor médio na população de peso estomacal próximo dos observados nos outros meses, o que significa que o percentual de repleção cheio e semi cheio individual nesse mês por ter sido mais alto que nos demais, evitou uma diferença na média populacional desse índice, como já observado na Figura 1.

A análise da dieta demonstrou a espécie Cyphocharax gilbert (Quoy \& Gaimard 1824), como presa preferencial da H. malabaricus no local de estudo, estando 
Citação: Beliene G. H., Rocha A. R. M. e Souza C. M. M., Parâmetros Alimentares de Hoplias malabaricus, como ferramenta de análise Ambiental na Lagoa Feia, RJ, Brasil. E\&S - Engineering and Science (2014) ,1:1.

presente em 65 (sessenta e cinco) dos Brycon opalinus (Cuvier 1819), com 2 (duas) estômagos analisados, seguido da espécie alem das espécies Rineloricaria sp1 e Geophagus brasiliensis (Quoy \& Gaimard Rineloricaria sp2 com 1 (uma) representação 1824) com 4 (quatro) representações e de cada (Tab. 2). Nessa mesma tabela observase que também foram encontrados 3 (três) estômagos com restos vegetais e 9 (nove) com conteúdo estomacal não identificado (NI), além dos estômagos vazios que aumentam gradativamente de novembro a junho e decrescendo no mês de julho somando-se ao fim do período de coleta 78 (setenta e oito).

\begin{tabular}{lccccccccc}
\hline & Nov & Dez & Fev & Mar & Abr & Mai & Jun & Jul \\
\hline Peso médio dos estômagos (g) & 30,8 & 18,9 & 23,0 & 19,6 & 13,9 & 20,7 & 17,1 & 12,7 \\
$\begin{array}{l}\mathbf{N}^{\mathbf{0}} \text { de estômagos cheios e semi } \\
\text { cheios }\end{array}$ & 16 & 12 & 11 & 11 & 9 & 10 & 5 & 8 \\
$\begin{array}{l}\text { Representação individual } \\
\text { estômagos cheio e semi cheio }\end{array}$ & de & $5,8 \%$ & $8,3 \%$ & $9,1 \%$ & $9,1 \%$ & $\begin{array}{c}11,1 \\
\%\end{array}$ & $10,0 \%$ & $20,0 \%$ & $\begin{array}{c}12,5 \\
\%\end{array}$ \\
\hline
\end{tabular}

Tabela 1: Valores mensais de peso médio de estômago em gramas, número de estômagos cheios e semi cheios e repressão individual de estômagos cheios e semi cheios

\begin{tabular}{lccccccccc}
\hline Itens alimentares & Nov & Dez & Fev & Mar & Abr & Mai & Jun & Jul & Total \\
C.gilbert & 12 & 9 & 10 & 10 & 5 & 8 & 4 & 7 & 65 \\
B. opalinus & 0 & 1 & 0 & 0 & 1 & 0 & 0 & 0 & 2 \\
Rineloricaria sp1 & 0 & 1 & 0 & 0 & 0 & 0 & 0 & 0 & 1 \\
Rineloricaria sp2 & 0 & 0 & 0 & 0 & 0 & 0 & 1 & 0 & 1 \\
G. brasiliensis & 0 & 0 & 1 & 1 & 1 & 0 & 0 & 1 & 4 \\
Restos vegetais & 0 & 0 & 1 & 1 & 1 & 0 & 0 & 0 & 3 \\
Vazios & 5 & 7 & 8 & 9 & 11 & 11 & 15 & 12 & 78 \\
NI* & 3 & 2 & 1 & 0 & 2 & 1 & 0 & 0 & 9 \\
\hline
\end{tabular}

$\mathrm{NI}^{*}=$ Não Identificado

Tabela 2: Freqüência dos itens alimentares observados nos estômagos dos espécimes de Hoplias malabaricus.

Os estados de repleção estomacal evidenciaram uma queda desde o início do estudo em torno de $70 \%$ cheio e $10 \%$ semicheio, até o mês de abril com $25 \%$ cheio e $20 \%$ semicheio, seguido por uma elevação no mês de maio com $45 \%$ cheio e $5 \%$ semicheio, declive acentuado em junho em $15 \%$ cheio e $10 \%$ semicheio voltando a subir no último mês para $35 \%$ cheio e $5 \%$ semicheio, (Figura 1). Esse comportamento de queda a partir do primeiro mês é comum e

evidencia a aproximação do período de seca, a partir de abril, tendência essa observada por vários autores, em diferentes épocas e locais (Winemiller 1989, Carvalho et al. 2002, Souza 2006, Corrêa \& Piedras 2009) nos períodos chuvosos. Além disso, durante estações quentes há a possibilidade do aumento da captura de presas relativas aos peixes piscívoros o que favorece a atividade de forrageamento (Menezes 1969), induzindo a uma queda na entrada das estações frias. Porém, durante essa estação fria e seca apesar de mais baixo a média de repleção se comparado ao período chuvoso, $(63 \%$ e $40 \%$ ), não é comum de se observar tanta variação nas taxas de captura de alimento, quando comparados a outros trabalhos já realizados com essa espécie de forma que por 
Citação: Beliene G. H., Rocha A. R. M. e Souza C. M. M., Parâmetros Alimentares de Hoplias malabaricus, como ferramenta de análise Ambiental na Lagoa Feia, RJ, Brasil. E\&S - Engineering and Science (2014) ,1:1.

algum motivo seja ele químico, físico ou biológico o ecossistema lagunar com toda sua rede intrínseca de relações tróficas deve estar garantindo essa relação alimentar alta entre a Hoplias malabaricus e suas presas, mesmo durante esse período de menores taxas alimentares. Essa alta oferta de alimento pode ser resultado de uma maior produção primárias nesse ambiente, que esteja sustentando as populações das presas da Hoplias que são caracteristicamente herbívoras, (Esteves \& Aranha 1999) oque leva a crer que possa estar ocorrendo entradas difusas ou pontuais de nutrientes alóctones nessa lagoa, certamente por via de efluentes domésticos e industriais, além do vinheto antes mencionado. Faz-se porém, necessárias constatações in situ desses indícios, que não foram possíveis de serem feitas nesse trabalho.

Além da característica observada anteriormente o mês de junho mesmo apresentando o menor valor dentre os estados de repleção observados não teve um reflexo tão acentuado na determinação do IRE, o que levou a uma análise individual do peso médio estomacal em cada mês, (Tab. 2), a fim de se estabelecer a percentagem média dos indivíduos com estômagos cheio e semi cheio. Os resultados demonstraram que o IRE desse mês não apresentou uma queda como a esperada em reflexo aos estados de repleção cheio e semi cheio devido ao fato de que os poucos indivíduos que se alimentaram, o fizeram com presas grandes, bem maiores que nos outros meses, conseguindo alcançar valores maiores que os esperados no gráfico de IRE. Esse comportamento mesmo que encerrado em apenas um dos meses de coleta, pode indicar algum ajuste ou mesmo mudança de comportamento alimentar mediante a dificuldade de adquirir alimentos, ou escassez desse, de forma a garantir a sobrevivência do animal através da captura de presas maiores e em menor quantidade. Begon et al.(2007) em seu livro Ecologia de indivíduos e ecossistemas, afirma que alguns carnívoros selecionam ativamente presas mais vantajosas em termos de ingestão de energia por unidade de tempo na captura delas, e que o alimento de um carnívoro freqüentemente varia pouco em composição mas pode variar quanto ao tamanho e a acessibilidade, reforçando a hipótese levantada. Já o fato das taxas de presas capturadas se baixa nesse mês pode estar relacionado ao fato de o mês de junho apresentar baixas taxas pluviométricas, oque levaria a uma diminuição do volume de água da lagoa e consequentemente a um aumento na concentração dos possíveis nutrientes citados anteriormente, oque iniciaria um processo de eutrofização e consequentemente baixaria as taxas de OD. Com isso ocorreria morte de uma considerada parcela dos organismos mais sensíveis a baixas concentrações de oxigênio, inclusive das espécies capturadas pela Hoplias.

Especificamente o IRE oscilou entre $6,14 \%$ e $4,07 \%$ até o mês de março quando e como já observado entre $5,06 \%$ e $2,99 \%$ de abril a julho final do levantamento. Os valores médios do índice para cada estação foram de $4,75 \%$ para estação chuvosa e quente e $4,23 \%$ para estação seca e fria, reforçando a tendência a homogeneidade desse índice alimentar entre as diferentes estações.

A análise da frequiência de itens alimentares surpreendeu pela presença de Cyphocharax gilbert como presa preferencial $(79,26 \%)$, em relação as diversas outras espécies comumente relatadas na dieta da Hoplias, que existem na Lagoa feia como mostra (Bizerril \& Primo, 2001), que foram encontradas respectivamente em 4,78\% ( $G$. brasiliensis); 2,43\% (B. opalinus); 1,21\% (Rineloricaria spl) e 1,21\% (Rineloricaria sp2), dos estômagos.

Devido ao fato da plasticidade trófica da Hoplias não ter se mostrado tão evidente esses resultados, levam a crer que possa existir uma relação alimentar intima entre essas espécies nesse ambiente específico ou que por algum motivo a predação de Cyphocharax gilbert esteja sendo facilitada, ou ainda que essa população esteja em maior abundancia em relação as demais que com ela ali coexistem e fazem parte da 
Citação: Beliene G. H., Rocha A. R. M. e Souza C. M. M., Parâmetros Alimentares de Hoplias malabaricus, como ferramenta de análise Ambiental na Lagoa Feia, RJ, Brasil. E\&S - Engineering and Science (2014) ,1:1.

alimentação da traíra, reforçando a hipótese de que esteja ocorrendo mudanças nos padrões tróficos desse ambiente em decorrência de fatores externos. Restos vegetais foram encontrados em 2 (dois) estômagos, porém não sendo considerados item da dieta da traíra, devido ao fato que provavelmente foram ingeridos acidentalmente durante uma investida contra alguma presa, como já foi relatado por alguns outros autores (Souza 2006, Corrêa \& Piedras 2009).

$\mathrm{O}$ aumento do número de estômagos vazios desde o primeiro mês de coleta até o início do período seco reforça a característica da Hoplias de diminuir seu ritmo de alimentação com a aproximação do inverno, porém, o comportamento observado durante esse mesmo período não se assemelha muito aos outros trabalhos sobre alimentação dessa espécie, levando a crer que fatores isolados ou trabalhando em conjunto estão facilitando ou dando condições de alimentação a Hoplias mesmo no período que normalmente relatam-se menores taxas de alimentação.

\section{CONCLUSÃO}

A partir desses resultados é possível inferir que a população de Hoplias malabaricus da Lagoa Feia apresentou pequenas diferenças alimentares como pontos importantes de partida para futuros estudos da espécie nesse local, ressaltando a biologia trófica que apontou para uma preferência alimentar pela espécie Cyphocharax gilbert, que por sua vez aparenta estar em grande abundância nesse ambiente aquático, denotando um comportamento alimentar anormal, mediante a plasticidade trófica observada em outros estudos em locais e tempos diferentes, e pelas altas taxas de alimentação observadas durante os meses frios, além da preferência por presas maiores no mês de menor incidência de capturas de presas, o qual pode indicar uma característica comportamental de sobrevivência mediante escassez de alimentos. Por outro lado esses mesmos dados indicam possíveis distúrbios que estejam afetando o arranjo da comunidade aquática da Lagoa Feia, ficando uma ressalva sobre os motivos que estão levando a esse comportamento, se são puramente naturais ou se estão sendo de alguma forma induzidos por ações humanas nesse ambiente.

\section{REFERÊNCIAS BIBLIOGRÁFICAS}

Amaral, Y.T; Souza, M.C, Novelli, R, Silva, H.J.T.; Almeida, M.G.; Souza, C.M.M. Biologia da traíira (hoplias malabaricus) na lagoa do campelo, RJ.. anais do IX congresso de ecologia do Brasil, 13 a 17 de setembro de 2009, São Lourenço - mg.

BEGON, M; Towsend, C.R; Harper, J.L. 2007. Ecologia: de indivíduos a ecossistemas. 4 ${ }^{\mathrm{a}}$. Edição. Porto Alegre, Artmed.

BIZERRIL, C. R. S. F.; PRIMO, P. B. S. Peixes de Águas Interiores do Estado do Rio de Janeiro. Rio de Janeiro: FEMARSEMADS, 2001.

Brum,W,M. et al. Condições organolépticas do pescado comercializado no município de Campos dos Goytacazes, RJ: dados preliminares. 2009.

CARNEIRO,P,R,F. Água e conflito na Baixada dos Goytacazes. REGA - Vol. 1, no. 2, p. 87-100, jul./dez. 2004.

Carvalho,L,N. Velasquez, $\quad$ C.H.F.; Moreira,V.S.S. Alimentação de Hoplias malabaricus (Bloch, 1794) (Osteichthyes, Erythrinidae) no rio Vermelho, Pantanal Sul Mato-Grossense. Universidade Federal de Mato Grosso do Sul, Rev. bras. Zoociências. 2002. p. 215-226.

Corrêa, F; Piedras,S,R,N. Feeding of Hoplias aff. malabaricus (Bloch, 1794) and Oligosarcus robustus Menezes, 1969 in a lagoon under estuarine influence, Pelotas, RS.Biotemas Year: 2009.

Costa, D,P,C. Martins, A.N; Sato, M.C.B. Metacercárias (Digenea, Diplostomidae) em Hoplias malabaricus (Actinopterygii, Erythrinidae) do reservatório de Três Marias, 
Citação: Beliene G. H., Rocha A. R. M. e Souza C. M. M., Parâmetros Alimentares de Hoplias malabaricus, como ferramenta de análise Ambiental na Lagoa Feia, RJ, Brasil. E\&S - Engineering and Science (2014) ,1:1.

alto rio São Francisco, Brasil. Revista Patologia Tropical, Volume 38, SUPL. 2 (jul-set) 2009.

ESTEVES K. E, ARANHA J. M.R. Trophic Ecology of stream fishes. Oecologia Australis, Vol 6 (1999)

Gimenes,M, F. Influência de variações morfológicas intraespecíficas no conteúdo energético de Hoplias aff. malabaricus (Bloch, 1794) na planície de inundação do alto rio Paraná. Dissertação de Mestrado apresentada ao programa de Pós-Graduação em Ecologia de Ambientes Aquáticos Continentais da Universidade Estadual de Maringá. PR, 2006.

Gonzaga,F,G. Simulação geoquímica 1D ao longo de uma seção geológica na Bacia de Campos [Rio de Janeiro] 2005. Universidade Federal do Rio de Janeiro, COPPE.2005.

Jacobina,U,P.Afonso, P.R.A.M, Carneiro, P.L.S, Dergan, J.A. .Biogeography and comparative cytogenetics between two populations of Hoplias malabaricus (Bloch, 1794) (Ostariophysi: Erythrinidae) from coastal basins in the State of Bahia, Brazil .Neotropical Ichthyology. Sociedade Brasileira de Ictiologia.2009.

Luiz,T,F. et al.Ecologia trófica da traira Hoplias malabaricus (BLOCH, 1794) introduzida no reservatório de Cachoeira Dourada - GO/MG. UFSCar, DHB, Laboratório de Dinâmica de Populações.2008.

MARTINS,J.M.E. Biologia de Hoplias malabaricus (Bloch, 1794) (Characiformes, Erythrinidae) na represa de Capim Branco I, Rio Araguari, MG. UFU. Uberlândia, MG. 71 p.2009.

PENTEADO,R,P. Bicho da vez $n^{o} 15$. Universidade Federal de Viçosa. Museu de
Zoologia João Moojen. Laboratório de Genética Ecológica e Evolutiva - UFV Campus Rio Paranaíba, 2009.

PETRY,A,C. A traíra Hoplias malabaricus (Bloch, 1794) na planície de inundação do alto rio Paraná: influência sobre as assembléias de peixes e aspectos da autoecologia . Universidade Estadual de Maringá. Maringá, PR. 2005.

REIS, R. E.; KULLANDER, S. O. \& FERRARIS, C. J., JR. 2003. Check list of the freshwater fishes of South and Central America. Porto Alegre, Edipucrs. 742p

SOUZA, M. C Avaliação de variáveis biológicas relevantes na distribuição d e metais pesados em tecido muscular de Hoplias malabaricus, (Bloch, 1974), Lagoa do Campelo, RJ. Campos dos Goytacazes, 2006. v, 66f.:Il

VAZZOLER, A.E.A .M.Biologia $d a$ reprodução de peixes teleósteos: teoria e prática. Maringá: EDUEM, 1996.196p.

Valpassos, C.A.M, Neto J. C. D. Diferentes percepções da natureza: As intervenções politécnicas, a fiscalização ambiental e pescadores artesanais na Lagoa Feia. Revista Anthropológicas, ano 10, volume 17(2): 95116 (2006)

VITORINO, C.A, SILVA, L.P, VENERE, P.C, SOUZA, I.L. Citogeografia dos peixes Erythrinídeos Hoplias Malabaricus (traíra) e Hoplerythrinus Unitaeniatus (jeju) do médio Araguaia. Universidade Federal de Mato Grosso, Instituto Universitário do Araguaia, Departamento de Ciências Biológicas e da Saúde. 2007.

Winemiller, K.O, Patterns of variation in life history among South American fishes in seasonal environments. O ecologia, 81: 225241.1989 